\title{
Constituyentes psicoactivos del cannabis y sus implicaciones clínicas: una revisión sistemática
}

\section{Psychoactive constituents of cannabis and their clinical implications: a systematic review}

\author{
C. Casajuana Kögel*****, H. López-Pelayo******, M. M. Balcells-Olivero***, \\ J. Colom****, A. GuAL*,***.
}

* Institut d'Investigacions Biomèdiques August Pi i Sunyer. ** Fundació Clínic per la Recerca Biomèdica. *** GRAC Addiction Unit Hospital Clínic of Barcelona. Red de Trastornos Adictivos (RTA). Universitat de Barcelona. **** Public Health Agency of Catalonia.

\section{Resumen}

Objetivo Esta revisión sistemática pretende resumir la actual evidencia sobre qué cannabinoides naturalmente presentes contribuyen a la psicoactividad final del cannabis, considerando sus concentraciones registradas y su farmacodinamia en humanos.

Metodología Siguiendo las guías PRISMA, se revisaron artículos científicos publicados antes de marzo 2016 en Medline, ScopusElsevier, Scopus, ISI-Web of Knowledge y COCHRANE, que cumplieran unos criterios establecidos a-priori.

Resultados En 40 artículos científicos, se identificaron tres cannabinoides naturalmente presentes $(\Delta-9$-Tetrahydrocannabinol, $\Delta$-8-Tetrahydrocannabinol y cannabinol) y un metabolito humano (11-OH-THC) con relevancia clínica. De éstos, el metabolito produce los efectos psicoactivos más potentes. El cannabidiol (CBD) no es psicoactivo, pero sí ejerce un efecto modulador sobre los efectos psicoactivos del cannabis. La concentración 9-THC en derivados cannábicos (hasta 40\%) supera en gran medida la de otros cannabinoides (hasta 9\%). La farmacodinamia descrita varía, dada la heterogeneidad en aspectos clave de la metodología (dosis, rutas de administración y experiencia previa con cannabis de los participantes). Conclusiones Los resultados evidencian que el 9-THC es el cannabinoide que más contribuye al efecto psicoactivo del cannabis. Otros cannabinoides psicoactivos contribuirían mínimamente, dada su menor potencia psicoactiva y su baja concentración en los derivados cannábicos. La falta de estándares metodológicos dificulta el avance en los conocimientos sobre los efectos del cannabis en la salud. Establecer una unidad estándar de cannabis basada en 9-THC ayudaría a superar estas limitaciones.

Palabras clave: Delta(9)-Tetrahidrocannabinol; Cannabinol; Cannabis; Cannabinoides; Drogas psicoactivas.

\begin{abstract}
Objective This systematic review aims to summarize current evidence on which naturally present cannabinoids contribute to cannabis psychoactivity, considering their reported concentrations and pharmacodynamics in humans.

Design Following PRISMA guidelines, papers published before March 2016 in Medline, Scopus-Elsevier, Scopus, ISI-Web of Knowledge and COCHRANE, and fulfilling established a-priori selection criteria have been included.

Results In 40 original papers, three naturally present cannabinoids ( $\Delta-9$ Tetrahydrocannabinol, $\Delta$-8-Tetrahydrocannabinol and Cannabinol) and one human metabolite (11-OH-THC) had clinical relevance. Of these, the metabolite produces the greatest psychoactive effects. Cannabidiol (CBD) is not psychoactive but plays a modulating role on cannabis psychoactive effects. The proportion of 9-THC in plant material is higher (up to $40 \%$ ) than in other cannabinoids (up to $9 \%$ ). Pharmacodynamic reports vary due to differences in methodological aspects (doses, administration route and volunteers' previous experience with cannabis).

Conclusions Findings reveal that 9-THC contributes the most to cannabis psychoactivity. Due to lower psychoactive potency and smaller proportions in plant material, other psychoactive cannabinoids have a weak influence on cannabis final effects. Current lack of standard methodology hinders homogenized research on cannabis health effects. Working on a standard cannabis unit considering 9-THC is recommended.

Keywords: Delta(9)-Tetrahydrocannabinol; Cannabinol; Cannabis; Cannabinoids; Psychotropic drugs.
\end{abstract}




\section{Introducción}

$\mathrm{E}$ 1 cannabis es la tercera droga más utilizada en todo el mundo (United Nations Office on Drugs and Crime, 2015), con prevalencia vitalicia estimada en unos 80,5 millones de los europeos (European Monitoring Centre for Drugs and Drug Addiction, 2012). Muchos países europeos informaron de un incremento en el consumo del cannabis durante las dos últimas décadas (OMS, 2016). Considerada la droga ilícita más ampliamente abusada, el Cannabis sativa (Cannabis Sativa L.) es una de las plantas más antiguamente cosechadas por el hombre (Appendino, Chianese, y Taglialatela-Scafati, 2011) y siempre se ha acompañado de controversia debido a sus efectos psicotrópicos—definidos por la OMS como la "capacidad de cambiar la conciencia, estado emocional o procesos del pensamiento de un individuo" (OMS, 2004).

El consumo de cannabis se ha asociado al deterioro psiquiátrico, físico y social (Hall y Degenhardt, 2009; Hall, 2009; Volkow, Baler, Compton, y Weiss, 2014). Sin embargo, se han encontrado varios potenciales efectos terapéuticos del cannabis (Mechoulam y Hanuš, 2000). Mientras que el conocimiento sobre el cannabis y su potencial terapéutico ha aumentado sensiblemente en las últimas décadas, su uso todavía es polémico debido a sus potenciales efectos nocivos y su marcado uso recreativo (Adams y Martin, 1996). Además, se han reportado las dificultades de separar los efectos psicotrópicos de los terapéuticos (Borgelt, Franson, Nussbaum, y Wang, 2013; Greenwald y Stitzer, 2000).

Una razón de esto es la composición compleja del cannabis, que contiene más de 500 compuestos de casi todas las clases químicas, como por ejemplo mono-y sesquiterpenos, azúcares, hidrocarburos, esteroides, flavonoides, compuestos nitrogenados y aminoácidos, ácidos grasos simples, entre otros (Appendino et al., 2011; ElSohly y Slade, 2005). Las fitocannabinoides son exclusivos del cannabis, siendo el Delta-9-Tetrahidrocannabinol (9-THC), el cannabinoide más estudiado debido a su conocida psicoactividad (Dewey, 1987; Gaoni y Mechoulam, 1964; Hollister, 1987). El resto de los cannabinoides, alrededor de 100, habitualmente se han desatendido (Mechoulam, 2005). Esto es especialmente preocupante ya que los consumidores mayormente fuman o ingieren derivados cannábicos, que presentan proporciones variables de cannabinoides.

Esta brecha en el conocimiento también ha complicado la evaluación de la salud acerca del cannabis. Como no existen sistemas fiables y homogéneos de registro, la evaluación del cannabis sigue centrándose en la frecuencia de consumo. Un ejemplo es la definición de los usuarios de cannabis en riesgo, dada por el EMCDDA, que se basa únicamente en la frecuencia de uso de cannabis en el último mes (European Monitoring Centre for Drugs and Drug Addiction, 2012). Mientras, la cantidad de cannabis consu- mida, y más concretamente la cantidad de los cannabinoides, permanece inexplorada.

Una opción ya definida para otras drogas, como el alcohol, son unidades estándar (Gual et al., 1999; Stockwell, Blaze-Temple, y Walker, 1991), que consideran el componente principal con implicación en la salud. Sin embargo, para el cannabis, se necesita un consenso sobre cuáles cannabinoides, además del 9-THC, pueden tener implicaciones sobre los efectos psicoactivos buscados en los seres humanos. Seguimos necesitando más información acerca de la influencia de otros cannabinoides sobre los efectos del cannabis, teniendo en cuenta sus concentraciones y sus efectos sobre la farmadinamia del cannabis.

Con el fin de analizar la contribución de otros cannabinoides a los efectos finales del cannabis en la salud, se realizó una revisión sistemática de la literatura, cuya finalidad es concluir qué cannabinoides naturalmente presentes han mostrado efectos psicoactivos en los seres humanos, teniendo en cuenta sus concentraciones y su farmadinamia.

\section{Métodos y materiales}

La información para esta revisión sistemática se recogió con un avanzado documento protocolo en concordancia con la línea directiva de PRISMA (Liberati et al., 2009; Urrútia y Bonfill, 2010). La búsqueda electrónica se realizó consultando con las siguientes cuatro bases de datos científicos: Medline (1950- March 2016), Scopus- Elsevier (2004March 2016), Web of Science (1900- March 2016) y COCHRANE (1991- March 2016). Se utilizó una combinación de los siguientes términos truncados como palabras clave para realizar la búsqueda: "Cannab*”, "marihuana", "hash", "química”, "estructura” "constituyente", "psico” y "efecto".

\section{Criterios de selección}

Se han tenido en cuenta todos los estudios publicados antes de octubre de 2015, siguiendo los parámetros: (1) Estudios sobre la psicoactividad en seres humanos con los cannabinoides naturalmente presentes en el cannabis o su alternativa sintética pura, (2) Las propiedades farmacodinámicas de los cannabinoides que contribuyen a los efectos psicoactivos finales del cannabis, (3) Informes sobre la potencia del cannabis. Criterios de exclusión: (1) Estudios centrados principalmente en las propiedades farmacocinéticas de los cannabinoides (2) Revisiones o monografías. No se aplicó ninguna restricción de lenguaje o fecha de publicación.

\section{Extracción de datos}

Dos revisores (CC y HL) extrajeron los datos y consultaron con dos investigadores de mayor categoría (AG y MB) en caso de dudas. De los artículos seleccionados, se extrajeron los siguientes datos: autoría, año de publicación, sustancias psicoactivas identificadas, dosis, formas de admi- 
nistración, efectos psicoactivos, derivados utilizados para el estudio y consumo previo de los voluntarios.

\section{Resultados}

Se encontró un total de 1484 entradas únicas, de los cuales 87 cumplieron nuestros criterios de inclusión. Después de la revisión del texto entero, se rechazaron 54 por cumplir los criterios de exclusión, sobre todo por ser revisiones anteriores $(\mathrm{N}=41)$. Por último, como se muestra en la Figure 1, se incluyeron 40 artículos en la revisión de la literatura. Los resultados se dividen en tres partes: 1) Los cannabinoides naturalmente presentes que afectan la psicoactividad del cannabis; 2) Efectos farmacodinámicos; y 3) las potencias informadas de los constituyentes psicoactivos naturalmente presentes.

\section{1) Cannabinoides naturalmente presentes que afectan la psicoactividad del cannabis}

\section{- Efectos directos}

Se observaron efectos psicoactivos dosis-dependientes del Delta-9-trans-Tetrahidrocannabinol (9-THC) en los sujetos después de usar las vías de administración intravenosa, oral e inhalada (Tabla 2). También se han reportado otras vías de administración como la vaporización (Zuurman et al., 2008) y beber té de cannabis (Hazekamp, Bastola, Rashidi, Bender, y Verpoorte, 2007).

Delta-8-Tetrahydrocannabinol (8-THC) y Cannabinol (CBN) producen efectos psicoactivos en los seres humanos pero con menor intensidad que el 9-THC (Tabla 1) (De Souza, Karniol, y Ventura, 1974; Hollister y Gillespie, 1973; Karniol y Carlini, 1973; Pérez-Reyes, 1973). Se estimó una proporción de potencia de 8-THC de entre 1:2 y 2:3
(8-THC: 9-THC) (Hollister y Gillespie, 1973; Karniol y Carlini, 1973). Se observaron efectos psicoactivos del 8-THC después de la administración intravenosa y oral. El CBN tiene una proporción de potencia de 1:10 (CBN: 9-THC), pero no se presentaron efectos psicoactivos después de la administración oral (Hollister, 1973).

Un metabolito de la 9-THC —el 11-OH-THC- tiene efectos psicoactivos por sí mismo si se inyecta puro por vía intravenosa, observando efectos psicoactivos más rápidos y más fuertes que después de la administración de 9-THC (Lemberger, Martz, Rodda, Forney, y Rowe, 1973).

\section{- Efectos indirectos}

La administración del cannabidiol (CBD) no fue seguida de efectos psicoactivos ni después la administración oral ni de la intravenosa. El CDB presenta un efecto modulador sobre la actividad psicoactiva del 9-THC, que ha demostrado depender de varios factores. Un ejemplo es la relación entre CBD:9-THC o el orden de administración de los cannabinoides, que influye en la intensidad del efecto de modulación (Dalton, Martz, Lemberger, Rodda, y Forney, 1976; Ilan, Gevins, Coleman, ElSohly, y de Wit, 2005; Zuardi, Shirakawa, Finkelfarb, y Karniol, 1982).

Otro cannabinoide que influye en los efectos psicoactivos del 9-THC es el $\Delta$-9-tetrahidrocannabivarin (THCV), cuya potencia se estimó en $25 \%$ de la potencia psicoactiva del 9-THC (Hollister, 1974). Sin embargo, la evidencia sobre los efectos del THCV sobre el 9-THC todavía es limitada y controvertida, sugiriendo que el THCV puede tener un efecto mixto en el 9-THC. Un estudio reciente demostró que el pre-tratamiento con THCV potenció algunos de los efectos producidos por el 9-THC, mientras que minimizó otros (Englund et al., 2016).

Tabla 1. La Psicoactividad de los cannabinoides naturalmente presentes y metabolitos relacionados en los seres humanos, identificadas en los artículos seleccionados.

\begin{tabular}{|c|c|c|c|}
\hline Molécula & Autor & Vía de administración & Potencia psicoactiva \\
\hline \multicolumn{4}{|c|}{ Cannabinoides psicoactivos naturalmente presentes } \\
\hline 9-THC & Todos autores & $\begin{array}{l}\text { Fumado } \\
\text { Intravenoso } \\
\text { Oral }\end{array}$ & $\begin{array}{l}\text { Cannabinoide naturalmente presente con la mayor potencia } \\
\text { psicoactiva }\end{array}$ \\
\hline \multirow[t]{2}{*}{ 8-THC } & Hollister (1973) & $\begin{array}{l}\text { Oral } \\
\text { Intravenoso }\end{array}$ & Proporción de potencia: 2:3 (8-THC : 9-THC) \\
\hline & $\begin{array}{l}\text { Karniol and Carlini (1973) } \\
\text { De Souza (1974) }\end{array}$ & Fumado & $\begin{array}{l}\text { Tasa de potencia: } 1: 2(8-\mathrm{THC}: 9-\mathrm{THC}) \\
\text { Menos potente que el 9-THC }\end{array}$ \\
\hline \multirow[t]{2}{*}{ CBN } & Pérez Reyes (1973) & Intravenoso & $1: 10(\mathrm{CBN}: 9-\mathrm{THC})$ \\
\hline & & Oral & Ningún efecto psicoactivo \\
\hline THCV & Hollister (1974) & Intravenoso & Proporción de potencia: 1:4 (THCV: 9-THC) \\
\hline
\end{tabular}

Metabolitos psicoactivos del cannabis directamente relacionados

11-OH-9-THC Lehmberger (1973) Intravenoso

Mayores efectos psicólogicos que el 9-THC con aparición más temprana

Nota. 9-THC: 9-Delta-tetrahidrocannabinol; 8-THC: 8-Delta-tetrahidrocannabinol; CBN: Cannabinol; THCV: $\triangle$-9-Tetrahidrocannabivarin; 11-OH-9-THC: 11-Hidroxi-Delta9-tetrahidrocannabinol. 
Tabla 2. Propiedades farmacodinámicos humanos de los cannabinoides descritos en los artículos seleccionados.

\begin{tabular}{|c|c|c|c|c|}
\hline Autor & $\begin{array}{l}\text { Características de los } \\
\text { voluntarios }\end{array}$ & $\begin{array}{l}\text { Vía de } \\
\text { administración }\end{array}$ & Dosis & Efectos observados después del consumo \\
\hline \multicolumn{5}{|c|}{ Administración de 9-THC puro } \\
\hline Curran (2002) & $\begin{array}{l}\text { Usuarios experimentados } \\
\text { de cannabis que no } \\
\text { consumen actualmente }\end{array}$ & Oral & 9-THC: $7,5,15 \mathrm{mg}$ & $\begin{array}{l}\text { Efectos dosis-dependiente en el deterioro de la } \\
\text { memoria episódica y el aprendizaje, el priming } \\
\text { perceptivo y la memoria de trabajo. }\end{array}$ \\
\hline $\begin{array}{l}\text { Martín-Santos } \\
(2012)\end{array}$ & $\begin{array}{l}\text { Uso de cannabis menos de } \\
15 \text { veces en la vida }\end{array}$ & Oral & 9-THC: $10 \mathrm{mg}$ & $\begin{array}{l}\text { Síntomas positivos y negativos como la ansiedad, } \\
\text { disforia, sedación e intoxicación subjetiva. El } 5 \% \text { de } \\
\text { los pacientes se volvió paranoide y ansioso. Aumento } \\
\text { de tasa cardíaca y diferencias en la presión arterial } \\
\text { diastólica a las } 2 \text { horas post-administración }\end{array}$ \\
\hline
\end{tabular}

Estudios con la administración de derivados cannábicos o combinaciones de cannabinoides

\begin{tabular}{llll}
\hline $\begin{array}{l}\text { Bhattacharyya } \\
(2010)\end{array}$ & $\begin{array}{l}\text { Media de consumo de } \\
\text { cannabis a lo largo de la } \\
\text { vida }\end{array}$ & $\begin{array}{l}\text { Por vía } \\
\text { intravenosa }\end{array}$ & $\begin{array}{l}\text { CBD: } 5 \mathrm{mg} \\
\text { 9-THC: } 1,25 \mathrm{mg}\end{array}$ \\
Dalton (1976) & $\begin{array}{l}\text { Usuarios previos de } \\
\text { cannabis }\end{array}$ & Fumado & $\begin{array}{l}25 \mu \mathrm{g} / \mathrm{kg} \text { of } 9-\mathrm{THC} \text { junto } \\
\text { con placebo o } 150 \mu \mathrm{g} / \mathrm{kg} \\
\text { de CBD }\end{array}$
\end{tabular}

$\begin{array}{llll}\begin{array}{l}\text { Greenwald } \\ (2000)\end{array} & \begin{array}{l}\text { Consumidores regulares de } \\ \text { marihuana }\end{array} & \begin{array}{l}\text { Fumado } \\ \text { (Marihuana) }\end{array} & \text { 9-THC: 3,55\% } \\ \text { Englund (2013) } & \begin{array}{l}\text { Voluntario habiendo } \\ \text { consumido al menos una } \\ \text { vez en su vida }\end{array} & \begin{array}{l}\text { Oral (CDB) } \\ \text { Intravenosa (9- }\end{array} & \begin{array}{l}\text { CBD 600 mg } \\ \text { THC) } 1,5 \mathrm{mg}\end{array} \\ \end{array}$

$\begin{array}{llll}\text { Englund (2016) } & \begin{array}{l}\text { Varones que no han } \\ \text { consumido cannabis más } \\ \text { de } 25 \text { veces en su vida }\end{array} & \begin{array}{l}\text { Oral (THCV) } \\ \text { Intravenosa } \\ \text { (THC) }\end{array} & \begin{array}{l}\text { THCV 10mg cápsulas } \\ 1 \mathrm{mg} \text { de 9-THC }\end{array} \\ \end{array}$

Haney (2015) Usuarios de cannabis de Oral (CDB) Pre-tratamiento con CDB al menos la mitad de un Fumado (THC) oral $(200 \mathrm{mg}, 400 \mathrm{mg} \mathrm{u}$ cigarrillo de cannabis 4 ó $\quad 800 \mathrm{mg}$ ) Fumar la mitad más veces por semana en el de un (5.30-5.80\% 9-THC) mes pasado cigarrillo de cannabis inactivo o activo, se fumó 90 min más tarde

$\begin{array}{llll}\text { Hunault (2008) } & \begin{array}{l}\text { Usuarios de cannabis (2-9 } \\ \text { porros/mes) }\end{array} & \begin{array}{l}\text { Fumado } \\ \text { (Marihuana y } \\ \text { tabaco) }\end{array} & \begin{array}{l}9-\text { THC: } 9,8 \%, 16,4 \%, \\ 23,1 \%\end{array} \\ \text { Hunault (2009) } & \begin{array}{l}\text { Usuarios de cannabis (2-9 } \\ \text { porros/mes) }\end{array} & \begin{array}{l}\text { Fumado } \\ \text { (Marihuana y } \\ \text { tabaco) }\end{array} & \begin{array}{l}9-\text { THC: } 9,8 \%, 16,4 \%, \\ 23,1 \%\end{array}\end{array}$

Pre-tratamiento con CDB dio lugar a la reducción de los efectos psicológicos/psicóticas del 9-THC versus pretratamiento con placebo.

La administración combinada de CBD y 9-THC produjo una respuesta subjetiva y sensaciones de intoxicación significativamente más atenuadas que después de la administración de 9-THC.

Pre-tratamiento con CBD no bloqueó la euforia inducida por el 9-THC.

Antinocicepción y síntomas comportamentales. Efectos subjetivos mostraron alta variabilidad entre participantes.

Pre-tratamiento con CBD produjo menos síntomas psicóticos, paranoia y mejor memoria episódica. Los síntomas psicóticos positivos eran menores con pretratamiento con CDB pero en comparación con placebo las diferencias no alcanzan significación estadística

Pre-tratamiento con THCV inhibió algunos efectos del THC (por ejemplo, efectos subjetivos del 9-THC menos intensos) mientras que potenció otros (efectos ansiógenos del 9-THC).

Pre-tratamiento oral con CDB no altera los efectos subjetivos, de refuerzo, o los efectos cardiovasculares de cannabis fumado comparado con placebo en fumadores de cannabis

Dosis crecientes aumentaron la tasa cardiaca y la somnolencia, produjo vómitos, cambios en la presión arterial y taquicardia.

Dosis crecientes retrasaron el tiempo de respuesta y empeoraron el control de manera lineal. Algunos participantes no mostraron deterioro en el control motor incluso con concentraciones séricas superiores a $40 \mathrm{ng} / \mathrm{mL}$. Los efectos subjetivos (sensación de estar "colocado" y somnolencia) variaban significativamente entre los tratamientos. 


\begin{tabular}{|c|c|c|c|c|}
\hline Autor & $\begin{array}{l}\text { Características de los } \\
\text { voluntarios }\end{array}$ & $\begin{array}{l}\text { Vía de } \\
\text { administración }\end{array}$ & Dosis & Efectos observados después del consumo \\
\hline Morgan (2010) & $\begin{array}{l}\text { Cannabis utilizado por lo } \\
\text { menos una vez al mes en el } \\
\text { último año }\end{array}$ & Fumado & Cannabis propio & $\begin{array}{l}\text { Déficits agudos en el recuerdo de prosa y deterioro } \\
\text { de la memoria, siendo más evidente si las } \\
\text { concentraciones de CBD eran bajas. }\end{array}$ \\
\hline Morgan (2012) & $\begin{array}{l}\text { Fumadores actuales de } \\
\text { cannabis }\end{array}$ & Fumado & Cannabis propio & $\begin{array}{l}\text { Altas concentraciones de THC aumentaron los índices } \\
\text { de depresión y ansiedad y produjeron peor recuerdo } \\
\text { de prosa y memoria de fuente. En los usuarios } \\
\text { recreacionales, la presencia de CBD disminuyó la } \\
\text { presencia de síntomas similares a la psicosis. En } \\
\text { usuarios diarios, la presencia de CDB dio lugar a mejor } \\
\text { memoria de reconocimiento. }\end{array}$ \\
\hline Ramesh (2013) & $\begin{array}{l}\text { Consumidores actuales de } \\
\text { marihuana a diario }\end{array}$ & Fumado & $\begin{array}{l}1 \text { a } 6 \text { caladas: } \\
\text { 9-THC: } 5,5 \%, 6,2 \%\end{array}$ & $\begin{array}{l}\text { Sensación de estar “colocado”, deterioro del } \\
\text { rendimiento psicomotor y disminución de la precisión } \\
\text { de la memoria inmediata. Participantes también } \\
\text { presentan disminución de niveles de monóxido de } \\
\text { carbono. }\end{array}$ \\
\hline Schaefer (1977) & $\begin{array}{l}\text { Fumadores ocasionales y } \\
\text { habituales }\end{array}$ & Fumado & 9- THC: $1,5 \%, 2,2 \%$ & $\begin{array}{l}\text { Aumento de la tasa cardiaca y tiempo de reacción más } \\
\text { lento. Efectos subjetivos eran dosis-dependientes. }\end{array}$ \\
\hline Schwope (2012) & $\begin{array}{l}\text { Fumadores de cannabis } \\
\text { intensos y crónicas }\end{array}$ & Fumado & 9- THC: $6,8 \%$ & $\begin{array}{l}\text { Sentimientos de sentirse “colocado", estimulación, } \\
\text { sedación, dificultad del habla, temblor. Aumento de } \\
\text { ingesta de comida y boca seca. }\end{array}$ \\
\hline Zuardi (1982) & $\begin{array}{l}\text { Use de cannabis por lo } \\
\text { menos en los } 15 \text { días antes } \\
\text { del estudio }\end{array}$ & Oral & $\begin{array}{l}\text { 9-THC: } 0,5 \mathrm{mg} / \mathrm{kg} \\
\text { CBD: } 1 \mathrm{mg} / \mathrm{kg} \\
\text { Combinación de 9-THC }(0,5 \\
\mathrm{mg} / \mathrm{kg}) \text { and CBD }(1 \mathrm{mg} / \mathrm{kg})\end{array}$ & $\begin{array}{l}\text { THC ansiogénico, el CBD antagonizó los efectos } \\
\text { psicotrópicos subjetivos del THC y sin afectar al pulso. }\end{array}$ \\
\hline
\end{tabular}

Estudios comparando la administración de 9-THC puro y derivados cannábicos

\begin{tabular}{|c|c|c|c|c|}
\hline Wachtel (2002) & $\begin{array}{l}\text { Consumo de cannabis al } \\
\text { menos una vez en los dos } \\
\text { últimos meses y por lo } \\
\text { menos } 10 \text { veces en su vida. }\end{array}$ & Oral y fumado & $\begin{array}{l}\text { 9-THC: } 8,4 \mathrm{mg}, 16,9 \mathrm{mg} \\
\text { CBN: } 0,30 \% \\
\text { CBD: } 0,05 \%\end{array}$ & $\begin{array}{l}\text { Grupo oral: voluntarios informan de mayores efectos } \\
\text { de la droga después del 9-THC puro que después de } \\
\text { tomar marihuana. } \\
\text { Grupo fumar: El 9-THC puro induce menos efectos de } \\
\text { droga que fumar marihuana, especialmente en la dosis } \\
\text { inferior. }\end{array}$ \\
\hline Chait (1992) & $\begin{array}{l}\text { Usuarios de cannabis } \\
\text { experimentados }\end{array}$ & Oral vs. fumado & $\begin{array}{l}\text { Oral: } 10 \mathrm{mg}, 15 \mathrm{mg} \\
\text { Fumado: } 2,6 \%, 3,6 \%\end{array}$ & $\begin{array}{l}\text { Fumar e ingestión oral produjeron efectos subjetivos } \\
\text { similares. Fumar marihuana produce mayores efectos } \\
\text { generales de droga, mayor tasa cardiaca y menos } \\
\text { ingestión de alimentos. }\end{array}$ \\
\hline Hart (2002) & $\begin{array}{l}\text { Fumadores de cannabis } \\
\text { actuales, con una media de } \\
6 \text { porros/día }\end{array}$ & Oral vs. fumado & $\begin{array}{l}\text { Oral: } 20 \mathrm{mg} \text { de } 9 \text {-THC } \\
\text { Fumado: } 3,1 \% \text { de } 9 \text {-THC }\end{array}$ & $\begin{array}{l}\text { Fumar e ingestión oral produjeron efectos subjetivos } \\
\text { similares. Después de fumar marihuana, se observaron } \\
\text { efectos subjetivos algo más pronunciados con } \\
\text { una disminución más lenta a lo largo del tiempo. } \\
\text { Se identificaron efectos subjetivos negativos y } \\
\text { abstinencia sólo en el grupo de fumadores y no en el } \\
\text { grupo de administración oral. }\end{array}$ \\
\hline
\end{tabular}

Nota. 9-THC: 9-Delta-tetrahidrocannabinol; 8-THC: 8-Delta-tetrahidrocannabinol ; CBN: Cannabinol; CBD: Cannabidiol; CBC: Cannabichromene.

\section{2) Efectos farmacodinámicos}

Los cannabinoides psicoactivos naturalmente presentes en el cánnabis han demostrado tener efectos farmacodinámicos tanto psicoloógicos como sistémicos (Tabla 2).

\section{- Medidas psicológicas}

El 9-THC puro y los derivados cannábicos producen efectos dosis-dependientes, siendo más frecuentes las descripciones de sensaciones de intoxicación y estimulación. Otros efectos frecuentemente observados fueron la ansiedad, la sedación, desviaciones del rendimiento psicomotor, deterioro de la memoria, peor recuerdo de prosa, cambios emocionales y precisión perceptiva disminuida (Curran,
Brignell, Fletcher, Middleton, y Henry, 2002; D’Souza et al., 2008; Hunault et al., 2009; Martin-Santos et al., 2012; Ramesh, Haney, y Cooper, 2013; Schaefer, Gunn, y Dubowski, 1977).

Los artículos que compararon los efectos del cannabis en las diferentes vías de administración (oral vs. fumado) concluyen que la administración oral de 9-THC puro produce valoraciones subjetivas más bajas que fumar derivados cannábicos (Chait y Zacny, 1992; Hart et al., 2002) y no se traduce en deseo y síntomas de abstinencia (Hart et al., 2002).

Al comparar los efectos del 9-THC puro y los derivados cannábicos dentro de la misma vía de administración, se observaron diferencias menores en los efectos subjetivos (Wachtel, ElSohly, Ross, Ambre, y Wit, 2002). Según esca- 
las analógicas visuales (Folstein y Luria, 1973), la marihuana ingerida por vía oral produce menos efectos subjetivos que el 9-THC puro oral. En cambio, cuando se fuma, la marihuana produjo mayores efectos subjetivos que fumar 9-THC puro. Estos resultados son consistentes con otros estudios que han mostrado que los cannabinoides como el CBN pierden sus efectos psicoactivos si se toman por vía oral (Pérez-Reyes, 1973).

Los estudios que analizaron la influencia de cannabinoides específicos en los efectos del 9-THC se centraron sobre todo en los CDB. Si bien desprovisto de psicoactividad (Pérez-Reyes, 1973), varios estudios incluidos en nuestra revisión sugieren que el CDB tiene una influencia potencial sobre los efectos finales del cannabis. Se ha mostrado que el CDB antagoniza y modula los efectos del 9-THC, como por ejemplo, el deterioro de la memoria y del recuerdo de la prosa (Morgan, Schafer, Freeman, y Curran, 2010). También parecen verse afectados la ansiedad y los síntomas similares a las psicóticas inducidos por el 9-THC si está presente el CDB (Morgan et al., 2012; Zuardi et al., 1982). Sin embargo, el rol del CDB parece complejo, ya que sus efectos no sólo dependen en su propia concentración sino también de la concentración del 9-THC, así como del orden de administración (Bhattacharyya et al., 2010; Ilan et al., 2005).

\section{- Efectos sistémicos}

Se encontraron efectos cardiovasculares dosis-dependientes, caracterizados por el marcado aumento de la tasa cardíaca en la mayoría de los voluntarios que participaron en estudios sobre cannabis. Fumar 9-THC puro indujo menos taquicardia que fumar los derivados cannábicos (Wachtel et al., 2002). Otros síntomas sistémicos eran la sensación de hambre (Schwope, Bosker, Ramaekers, Gorelick, y Huestis, 2012), disminución del equilibrio y aumento del tamaño de las pupilas (Zuurman et al., 2008).

No se describieron efectos sobre el sistema respiratorio en los artículos seleccionados. Dado que el cannabis habitualmente se fuma junto con tabaco, algunos estudios analizaron la potencial interacción entre las dos drogas. Si bien es escasa, la información disponible indica que el tabaco aumenta la proporción de los cannabinoides liberados (Van der Kooy, Pomahacova, y Verpoorte, 2009).

\section{3) Potencias reportadas de los constituyentes psi- coactivos naturalmente presentes}

\section{- 9-THC}

Con unas pocas excepciones, el 9-THC es el cannabinoide presente en mayor proporción. Las mayores concentraciones de 9-THC identificadas en la revisión fueron el polvo de cannabis inglés $(40,63 \%)$ y el hachís holandés $(39,85 \%)$. Se ha informado de concentraciones inferiores de 9-THC en el cannabis herbal, con un máximo de
25,5\% de 9-THC encontrado en Nueva Zelanda. Estudios que analizan cambios en las concentraciones de 9-THC a lo largo del tiempo describen grandes aumentos en la proporción del principal cannabinoide psicoactivo (Bruci et al., 2012; Burgdorf, Kilmer, y Pacula, 2011; ElSohly et al., 2000, 2016; Mehmedic et al., 2010) (Tabla 3).

\section{- Otros cannabinoides que contribuyen a la psicoactividad del} cannabis

No siempre se registraron concentraciones de cannabinoides psicoactivos diferentes del 9-THC (Tabla 3). Cuando están presentes, las concentraciones generalmente fueron bajas en comparación con el 9-THC. Un ejemplo es el CBN, cuya máxima concentración registrada fue de 7,7\%, presente en aceite de hachís confiscado en EEUU (Mehmedic et al., 2010).

En cambio, si bien no psicoactivas, las concentraciones de CBD se registraron con frecuencia. A lo largo del tiempo, los porcentajes de CBD en el cannabis muestran una tendencia negativa, que es especialmente visible en el cannabis herbal (ElSohly et al., 2016; Mehmedic et al., 2010; Niesink, Rigter, Koeter, y Brunt, 2015; Potter, Clark, y Brown, 2008). En el cannabis de resina se encontraron potencias variables dependiendo del origen del derivado (Niesink et al., 2015; Pijlman, Rigter, Hoek, Goldschmidt, y Niesink, 2005; Tsumura et al., 2012).

\section{Discusión}

Nuestra revisión resume la actual evidencia sobre la contribución de los cannabinoides naturalmente presentes a los efectos psicoactivos finales del cannabis. Hemos identificado tres cannabinoides (9-THC, 8-THC y CBD) y un metabolito humano del 9-THC (11-OH-THC) que han mostrado efectos psicoactivos. Más allá de los constituyentes psicoactivos naturalmente presentes, el 9-THC presenta los efectos psicoactivos más fuertes y está presente en la concentración más alta. Su metabolito 11-OH-THC produce efectos más intensos que aparecen antes. Los efectos psicológicos y sistémicos del cannabis son mayormente inducidos por el 9-THC, mientras que la contribución de otros cannabinoides psicoactivos es estimada como muy baja.

\section{Limitaciones en los informes farmacodinámicos del cannabis}

Los estudios incluidos presentan grandes diferencias en aspectos cruciales de la metodología, lo que dificulta la comparación directa y un análisis más exhaustivo, como por ejemplo un meta-análisis. Uno de estos aspectos es la experiencia previa de los voluntarios, que variaba mucho (pasando de un consumo de cannabis de más de 10 veces en la vida a usuarios crónicos e intensos de cannabis). Al estudiar la farmadinamia del cannabis, la experiencia previa es determinante para estimar los efectos agudos y a lar- 
Tabla 3. Registros de concentraciones de cannabinoides en los artículos seleccionados.

\begin{tabular}{|c|c|c|c|c|c|c|c|c|}
\hline Autor & Origen y año & Tipo de derivado & \multicolumn{6}{|c|}{ Concentraciones registradas de cannabinoides analizados } \\
\hline $\begin{array}{l}\text { Bruci et al } \\
(2012)\end{array}$ & Albania, 2011 & Herbal & \multicolumn{6}{|c|}{$\begin{array}{l}\text { 9-THC: } 1,07 \%-12,13 \% \\
\text { CBD: } 0,65 \%-2,02 \% \\
\text { CBN: } 0,02 \%-1,12 \%\end{array}$} \\
\hline $\begin{array}{l}\text { Burgdorf et al } \\
\text { (2011) }\end{array}$ & $\begin{array}{l}\text { USA; 1996- } \\
2008\end{array}$ & No se indica & \multicolumn{6}{|c|}{$\begin{array}{l}\text { 9-THC: } 11,75 \% \\
\text { CBD: } 0,08 \%\end{array}$} \\
\hline ElSohly (2000) & $\begin{array}{l}\text { USA, 1980- } \\
1997\end{array}$ & $\begin{array}{l}\text { Cannabis, hachís, o } \\
\text { aceite de hash }\end{array}$ & \multicolumn{6}{|c|}{$\begin{array}{l}\text { Las muestras de marihuana tenían menos del 1,5\% de 9-THC en } 1980 \text { y ascendió a 4,2\% } \\
\text { de } 9 \text {-THC en } 1997 . \text { Hachís y aceite de hash no mostraron tendencias específicas de } \\
\text { potencia. Otros cannabinoids, CBD, CBN y CBC no mostraron cambios significativos en su } \\
\text { concentración a lo largo de los años. }\end{array}$} \\
\hline ElSohly (2016) & $\begin{array}{l}\text { USA, 1995- } \\
2014\end{array}$ & $\begin{array}{l}\text { Marihuana, hachís, o } \\
\text { aceite de hash }\end{array}$ & \multicolumn{6}{|c|}{$\begin{array}{l}\text { La potencia de } 9 \text {-THC en el cannabis herbal ha aumentado a lo largo del tiempo desde } \\
\text { aproximadamente } 4 \% \text { de } 9 \text {-THC en } 1995 \text { a aproximadamente } 12 \% \text { en } 2014 \text {. Otros } \\
\text { cannabinoides con contenido significativo son el CBD y el CBN (en aceite de hachís } \\
\text { aproximadamente } 2-5 \% \text { ). El contenido en CBD en derivados cannábicos ha caído desde } \\
\text { aproximadamente } 0,28 \% \text { en } 2001 \text { a }<0,15 \% \text { en } 2014 \text {. Se mantiene un promedio de CBD por } \\
\text { debajo del } 5 \% \text { en el derivado de resina. }\end{array}$} \\
\hline \multirow[t]{7}{*}{$\begin{array}{l}\text { Mehmedic } \\
(2010)\end{array}$} & \multirow[t]{7}{*}{$\begin{array}{l}\text { USA, 1993- } \\
2008\end{array}$} & \multirow{7}{*}{$\begin{array}{l}\text { Marihuana } \\
\text { Sinsemilla } \\
\text { Hierba de la cuneta } \\
\text { Hachís } \\
\text { Aceite de hash }\end{array}$} & $\%$ & Marihuana & Sinsemilla & $\begin{array}{l}\text { Hierba de la } \\
\text { cuneta }\end{array}$ & Hachís & $\begin{array}{l}\text { Aceite de } \\
\text { hash }\end{array}$ \\
\hline & & & 9-THC & $3,4-6,1$ & $5,8-13,4$ & $0,3-0,5$ & $2,5-29,3$ & $6,5-31,5$ \\
\hline & & & CBD & $0,2-0,5$ & $0,2-0,5$ & $1,5-2,4$ & $0,8-4,9$ & $0,1-1,3$ \\
\hline & & & CBN & $0,2-0,4$ & $0-0,2$ & $0-0,2$ & $1,3-2,3$ & $0,6-7,7$ \\
\hline & & & $\mathrm{CBC}$ & $0,2-0,3$ & $0,2-0,3$ & $0,1-0,2$ & $0,5-0,9$ & $0,3-1,6$ \\
\hline & & & CBG & $0,1-0,3$ & $0,1-0,5$ & $<0,1$ & $0,3-1$ & $0,2-1,2$ \\
\hline & & & THCV & $<0,1$ & $<0,1$ & $<0,1$ & $0,1-0,4$ & $0,1-0,7$ \\
\hline Niesink (2015) & $\begin{array}{l}\text { Países Bajos, } \\
\text { 2005-2015 }\end{array}$ & $\begin{array}{l}\text { Cannabis herbal } \\
\text { (Nederwiet y hierba de } \\
\text { cannabis importada), } \\
\text { resina de cannabis } \\
\text { (Nederhasj y resina de } \\
\text { cannabis importada) }\end{array}$ & \multicolumn{6}{|c|}{$\begin{array}{l}\text { Cannabis herbal: Nederwiet mostró altas dosis de THC pero apenas CDB; menos del } 1 \% \\
\text { de estas muestras contenía más de } 1 \% \text { de CBD. Las potencias medias del Nederwiet más } \\
\text { popular y más fuerte eran de } 16,0 \pm 4,0 \%, 17,0 \pm 3,9 \% \text {. El cannabis herbal tenía potencias } \\
\text { menores de } 9 \text {-THC }(6,5 \pm 3,5 \%) \text {. La resina de cannabis importada tenía } 16,5 \pm 6,3 \% \text { y el } \\
\text { Nederhasj presentaba niveles superiores de } 9 \text {-THC }(30,2 \pm 16,4 \%) \text {. }\end{array}$} \\
\hline $\begin{array}{l}\text { van der Pol } \\
(2013)\end{array}$ & $\begin{array}{l}\text { Países Bajos, } \\
\text { año no } \\
\text { indicado }\end{array}$ & $\begin{array}{l}\text { Porros de cannabis } \\
\text { herbal y resina de } \\
\text { cannabis }\end{array}$ & \multicolumn{6}{|c|}{$\begin{array}{l}\text { Herbal: 9-THC: } 12,4 \%(\text { rango } 1,1-19,5, \mathrm{DT}=3,0) ; \mathrm{CDB}: 0,2 \% \text { (rango: } 0,0-0,5, \mathrm{DT}=0,1) \\
\text { Resina: } 9-\mathrm{THC}: 12,2 \% \text { (rango 2,9-24,7, DT=6.0); CDB: } 5,4 \% \text { (rango: } 0,2-7,9, \mathrm{DT}=1,5 \text { ) }\end{array}$} \\
\hline \multirow[t]{4}{*}{ Pijlman (2005) } & \multirow[t]{4}{*}{$\begin{array}{l}\text { Países Bajos, } \\
\text { 2000-2004 }\end{array}$} & \multirow{4}{*}{$\begin{array}{l}\text { Marihuana importada, } \\
\text { cosecha propia } \\
\text { marihuana, hachís } \\
\text { importado y cosecha } \\
\text { propia hachís }\end{array}$} & $\%$ & $\begin{array}{l}\text { Marihuana } \\
\text { importada }\end{array}$ & $\begin{array}{l}\text { Cosecha } \\
\text { propia Marih. }\end{array}$ & $\begin{array}{l}\text { Hachís } \\
\text { importado }\end{array}$ & $\begin{array}{l}\text { Cosecha } \\
\text { propia Ha }\end{array}$ & \\
\hline & & & 9-THC & 7,2 & 21,5 & 18,5 & 39,8 & \\
\hline & & & CBN & 0,7 & 0 & 1,5 & 0,6 & \\
\hline & & & CBD & 0,20 & 0,25 & 8,10 & 0,60 & \\
\hline \multirow[t]{7}{*}{ Potter (2008) } & \multirow{7}{*}{$\begin{array}{l}\text { Inglaterra, } \\
2005\end{array}$} & \multirow{7}{*}{$\begin{array}{l}\text { Resina } \\
\text { Herbal } \\
\text { Sinsemilla } \\
\text { Polvo }\end{array}$} & $\%$ & Herbal & Resina & Sinsemilla & Polvo & \\
\hline & & & 9-THC & 2,14 & 3,54 & 13,98 & 40,63 & \\
\hline & & & CBD & $<0,1$ & 4,17 & $<0,10$ & 0,18 & \\
\hline & & & $\mathrm{CBC}$ & 0,22 & 0,34 & 0,2 & 0,41 & \\
\hline & & & THCV & 0,17 & 0,10 & $<0,03$ & 0,29 & \\
\hline & & & CBG & 0,21 & 0,29 & 0,41 & 1,59 & \\
\hline & & & CBN & 0,55 & 1,55 & 0,16 & 0,57 & \\
\hline \multirow[t]{4}{*}{$\begin{array}{l}\text { Tsumura } \\
(2012)\end{array}$} & \multirow[t]{4}{*}{$\begin{array}{l}\text { Japón, 2010- } \\
2011\end{array}$} & \multirow{4}{*}{$\begin{array}{l}\text { Hojas } \\
\text { Brotes con semillas } \\
\text { Brotes sin semillas } \\
\text { Polvo }\end{array}$} & $\%$ & Hojas & $\begin{array}{l}\text { Brotes } \\
\text { con semillas }\end{array}$ & $\begin{array}{l}\text { Brotes } \\
\text { sin semillas }\end{array}$ & Polvo & \\
\hline & & & 9-THC & 1,8 & 3,8 & 8,3 & 8,9 & \\
\hline & & & CBN & 0,5 & 1,1 & 1,3 & 1,2 & \\
\hline & & & CBD & 0,2 & 0,2 & 0,1 & 0,1 & \\
\hline Turner (1974) & $\begin{array}{l}\text { Diferentes } \\
\text { orígenes, } \\
\text { década de } \\
1970\end{array}$ & Plantas de cannabis & $\begin{array}{l}\text { Nepal: } \\
\text { Méjico } \\
\text { Paquis } \\
\text { USA: } 0 \\
\text { Otros l }\end{array}$ & $\begin{array}{l}\text { THC, } 0,21 \% \text { C } \\
\text { THC, } 0,27 \% \\
0 \% \text { THC, } 1,14 \\
\text { IC, } 1,42 \% \text { CB } \\
\text { abinoides en }\end{array}$ & CBD & BC, THCV y CB & & \\
\hline
\end{tabular}

Nota. 9-THC: 9-Delta-tetrahidrocannabinol; CBN: Cannabinol; CBD: Cannabidiol; CBC: Cannabichromene; CBG: cannabigerol; THCV: Tetrahidrocannabivarin; CBL: Cannabicyclol. 
go plazo, debido a la presencia de los niveles de depósito y de tolerancia (Abood y Martin, 1992; De Souza et al., 1974; Sharma, Murthy, y Bharath, 2012). Las diferencias metodológicas también afectan la medición de los resultados, pasando de auto-evaluaciones subjetivas de efectos similares a la marihuana en los estudios más antiguos (Pérez-Reyes, 1973) a descripciones mucho más complejas usando escalas validadas, en los artículos más recientes recuperados (Englund et al., 2016).

Otro aspecto importante a considerar es la vía de administración. Las diferencias juegan un papel clave en los efectos del cannabis, con un impacto directo en los cannabinoides farmacocinéticos. Un ejemplo es el CBN, que produce efectos psicoactivos si se inyecta por vía intravenosa pero no por administración oral (Pérez-Reyes, 1973). Otro ejemplo son los síntomas de abstinencia, que aparecieron después de fumar marihuana pero no después de la ingestión oral de 9-THC puro (Hart et al., 2002). Como habitualmente se fuma el extracto de cannabis, estos estudios tienen implicaciones importantes para la evaluación de los efectos del cannabis en la salud.

Además, las dosis de 9-THC en los artículos seleccionados difieren ampliamente. En consecuencia, como el cannabis tiene efectos dosis-dependientes (D'Souza et al., 2008; Wachtel et al., 2002), se describieron síntomas variables. No podemos rechazar que, debido a la composición compleja del cannabis, incluso los derivados cannábicos que contienen niveles similares de 9-THC pueden haber sido diferentes con respecto a otros cannabinoides. Las características como el origen de la materia vegetal, la parte analizada o el producto derivado del cannabis siempre deben considerarse. Además, con el fin de permitir comparaciones entre estudios, también los datos sobre otros cannabinoides distintos del 9-THC deben registrarse.

\section{Evaluación del rol de otros cannabinoides distintos del 9-THC}

Nuestra revisión pone de relieve que, por ahora, se considera el 9-THC como el cannabinoide principal responsable de los efectos psicoactivos del cannabis. Las investigaciones se han centrado en el 9-THC, si bien el cannabis se consume sobre todo en forma de derivado cannábico. Con el fin de evitar informes farmacológicos contradictorios, varios autores han indicado que otros cannabinoides herbales deben considerarse al evaluar los efectos del cannabis (Eichler et al., 2012; Mechoulam, 2005; Turner, 1974). Nuestra revisión muestra que, en la mayoría de las ocasiones, ni siquiera se analizan otros los cannabinoides psicoactivos.

Se podría encontrar un perfil común de los efectos del cannabis, incluyendo efectos subjetivos (sentirse colocado, estimulado) y efectos sistémicos (cambios en la tasa cardiaca). Estudios que han investigado si el 9-THC es el único responsable de la psicoactividad del cannabis concluyen que la administración de 9-THC puro y de derivados can- nábicos producen efectos similares que no difieren significativamente (Ilan et al., 2005; Wachtel et al., 2002). Las implicaciones clínicas debidas a la interacción del 9-THC con el CBD todavía están siendo disputadas, y los artículos incluidos muestran que los efectos agudos de modulación del CDB dependen de varios factores, como, por ejemplo, la proporción de la concentración, la forma de administración o el orden de administración (Englund et al., 2013; Haney et al., 2016; Ilan et al., 2005; Zuardi et al., 1982). Los efectos descritos reflejan las condiciones de laboratorio, que pueden diferir de las condiciones de la vida real. Es más, los datos sobre las concentraciones de CBD indican que, especialmente en el cannabis herbal, el CBD sólo está presente en concentraciones menores. Por ello y como se ha mencionado en revisiones anteriores sobre la interacción del CBD y el 9-THC, la evidencia sugiere que las implicaciones clínicas del CBD sobre los resultados del cannabis con respecto a la salud requieren más investigación, que incluya muestras mas grandes y análisis de los efectos a largo plazo (Haney et al., 2016; Hollister y Gillespie, 1975; Leweke, Mueller, Lange, y Rohleder, 2016; Zhornitsky y Potvin, 2012).

\section{Potencias de cannabis e implicaciones para la salud}

En nuestra selección de artículos, la concentración más alta utilizada para analizar los efectos farmacodinámicos fue de 69 mg de 9-THC (23\%) (Hunault et al., 2008, 2009), aunque las altas concentraciones utilizadas en la investigación pueden ser inferiores que algunas de las potencias registradas, como las muestras que contenían entre $30 \%$ y $40 \%$ de 9-THC que fueron reportadas por varios autores. Sin embargo, debido al hecho de que algunos registros corresponden a decomisos policíacos, los datos sobre la potencia pueden no ser representantes del cannabis común de la calle.

Algunos autores afirman que, cuando se cultiva para uso recreativo, conseguir cannabis más fuerte se ha convertido en una meta habitual (Knight et al., 2010; Mehmedic et al., 2010; Pijlman et al., 2005). Los cambios en la concentración se han centrado en el 9-THC, mientras que otros cannabinoides se mantienen o disminuyen (Mehmedic et al., 2010). La mayoría de las concentraciones de 9-THC eran superiores a las concentraciones de otros cannabinoides, que mayormente no superan el $8 \%$.

Se requiere más información acerca de cuáles de los efectos nocivos del cannabis se agravarían con dosis superiores, especialmente en casos de uso crónico e intenso. Sin embargo, nuestra revisión muestra que hay una tendencia a simular las condiciones reales del consumo de cannabis en las investigaciones. Un ejemplo es el análisis de extractos de cannabis mezclados con tabaco (Hunault et al., 2008, 2009; Van der Kooy, Pomahacova, y Verpoorte, 2008; Van der Kooy et al., 2009) o los preparados de los propios voluntarios (Morgan et al., 2012; van der Pol et al., 2013). 
Otra cuestión de creciente preocupación es el uso de cannabinoides sintéticos muy potentes, que pueden ocasionar efectos nocivos graves en la salud. Nuestra revisión no consideró estos compuestos debido al hecho de que su prevalencia de uso en nuestro contexto es muy inferior al uso de los derivados (Observatorio Español de la Droga y las Toxicomanías, 2015; Plan Nacional sobre Drogas, 2016).

\section{Limitaciones y puntos fuertes de la revisión sistemática}

Nuestra revisión tiene varias limitaciones potenciales. Por un lado, las diferencias en las características de los estudios dificultaban la extracción equivalente de datos para su comparación en un meta-análisis. Por otro lado, el sesgo de publicación y la limitación de las bases de datos pueden haber producido algún tipo de pérdida de información. Sin embargo, nuestra revisión se diseñó con el fin de encontrar y evaluar estudios relevantes o de calidad que abordasen el tema.

Nuestra revisión tiene varios aspectos positivos. Que sepamos, es la primera revisión sistemática de la literatura que se centra en la psicoactividad, considerando las propiedades farmacodinámicas y las potencias de varios cannabinoides. Nuestro estudio también señala que varios aspectos de la psicoactividad del cannabis aún son poco claros, sobre todo porque la investigación no se ha centrado en cómo los cannabinoides pueden influir de forma individual en los efectos finales del cannabis.

\section{Conclusiones}

La evidencia actual indica que, de los constituyentes naturalmente presentes en el cannabis, el 9-THC es el cannabinoide psicoactivo más potente. Además, en comparación con otros cannabinoides, su concentración en los derivados es muy superior. Por ello, al evaluar los efectos del cannabis, el 9-THC se debe considerar como el contribuyente principal a la psicoactividad del cannabis.

El cannabis es la droga ilícita más abusada en todo el mundo y constituye un importante problema de salud pública. Se requiere metodología estandarizada para superar las limitaciones actuales en la investigación del cannabis. Se requiere trabajar con una unidad estándar de cannabis que cuantifique el principal cannabinoide del cannabis con implicación en la psicoactividad. Se espera que esta unidad facilite la homogeneización de los registros del cannabis, que es fundamental para mejorar la investigación epidemiológica y las intervenciones de salud pública.

\section{Aportaciones}

Cristina Casajuana Kögel, Hugo López-Pelayo, María Mercedes Balcells y Antoni Gual diseñaron el estudio. Cristina Casajuana hizo la primera redacción del manuscrito.
Todos los demás autores contribuyeron a la edición y revisión final del manuscrito. Todos los autores aprobaron el documento final.

\section{Agradecimientos}

Ministerio de Sanidad y Consumo (PNSD 2013I082), Fundació Clínic per la Recerca Biomèdica, IDIBAPS y Generalitat de Catalunya. Las conclusiones del artículo son únicamente responsabilidad de los autores y no necesariamente representan los puntos de vista oficiales de las instituciones, que no tenían ningún otro papel en el diseño del estudio, recogida, análisis, e interpretación de los datos; en la redacción del artículo; o en la decisión de presentar el artículo para su publicación.

\section{Declaración}

Hugo López-Pelayo ha recibido becas de viaje de los laboratorios Lundbeck, Lilly, Pfizer, Rovi, Esteve y honorarios y becas de viaje de Janssen. Antoni Gual ha recibido becas honorarias, ayudas a la investigación y becas de viaje de Lundbeck, Janssen, Pfizer, Lilly, Abbvie D\&A Pharma, y Servier. Los demás autores declaran no tener ningún conflicto potencial de intereses.

\section{References}

Abood, M. E. y Martin, B. R. (1992). Neurobiology of marijuana abuse. Trends in Pharmacological Sciences, 13, 201206. doi:10.1016/0165-6147(92)90064-D.

Adams, I. B. y Martin, B. R. (1996). Cannabis: pharmacology and toxicology in animals and humans. Addiction, 91, 1585-1614. doi:10.1046/j.1360-0443.1996.911115852.x.

Appendino, G., Chianese, G. y Taglialatela-Scafati, O. (2011). Cannabinoids: Occurrence and Medicinal Chemistry. Current Medicinal Chemistry, 18, 1085-1099. doi: 10.2174/092986711794940888.

Bhattacharyya, S., Morrison, P. D., Fusar-Poli, P., Martin-Santos, R., Borgwardt, S., Winton-Brown, T., ... McGuire, P. K. (2010). Opposite Effects of $\delta-9$ Tetrahydrocannabinol and Cannabidiol on Human Brain Function and Psychopathology. Neuropsychopharmacology, 35, 764-774. doi:10.1038/npp.2009.184.

Borgelt, L. M., Franson, K. L., Nussbaum, A. M. y Wang, G. S. (2013). The Pharmacologic and Clinical Effects of Medical Cannabis. Pharmacotherapy: The Journal of Human Pharmacology and Drug Therapy, 33, 195-209. doi:10.1002/phar.1187.

Bruci, Z., Papoutsis, I., Athanaselis, S., Nikolaou, P., Pazari, E., Spiliopoulou, C. y Vyshka, G. (2012). First systematic evaluation of the potency of Cannabis sativa plants grown in Albania. Forensic Science International, 222, 4046. doi:10.1016/j.forsciint.2012.04.032. 
Burgdorf, J. R., Kilmer, B. y Pacula, R. L. (2011). Heterogeneity in the composition of marijuana seized in California. Drug and Alcohol Dependence, 117, 59-61. doi:10.1016/j.drugalcdep.2010.11.031.

Chait, L. D. y Zacny, J. P. (1992). Reinforcing and subjective effects of oral 89 -THC and smoked marijuana in humans. Psychopharmacology, 107, 255-262. doi:10.1007/ BF02245145.

Curran, V., Brignell, C., Fletcher, S., Middleton, P. y Henry, J. (2002). Cognitive and subjective dose-response effects of acute oral $\delta 9$-tetrahydrocannabinol (THC) in infrequent cannabis users. Psychopharmacology, 164, 61-70. doi:10.1007/s00213-002-1169-0.

D’Souza, D. C., Ranganathan, M., Braley, G., Gueorguieva, R., Zimolo, Z., Cooper, T., ... Krystal, J. (2008). Blunted psychotomimetic and amnestic effects of delta-9-tetrahydrocannabinol in frequent users of cannabis. Neuropsychopharmacology, 33, 2505-2516. doi:10.1038/sj.npp.1301643.

Dalton, W. S., Martz, R., Lemberger, L., Rodda, B. E. y Forney, R. B. (1976). Influence of cannabidiol on delta-9-tetrahydrocannabinol effects. Clinical Pharmacology y Therapeutics, 19, 300-309. doi:10.1002/cpt1976193300.

De Souza, M. R. C., Karniol, I. G. y Ventura, D. F. (1974). Human tonal preferences as a function of frequency under 88-tetrahydrocannabinol. Pharmacology Biochemistry and Behavior, 2, 607-611. doi:10.1016/00913057(74)90028-8.

Dewey, W. L. (1987). Cannabinoid pharmacology. Journal of Ethnopharmacology, 20, 293-294. doi:10.1016/03788741(87)90061-4.

Eichler, M., Spinedi, L., Unfer-Grauwiler, S., Bodmer, M., Surber, C., Luedi, M. y Drewe, J. (2012). Heat Exposure of Cannabis sativa Extracts Affects the Pharmacokinetic and Metabolic Profile in Healthy Male Subjects. Planta Medica, 78, 686-691. doi:10.1055/s-0031-1298334.

ElSohly, M. A., Mehmedic, Z., Foster, S., Gon, C., Chandra, S. y Church, J. C. (2016). Changes in Cannabis Potency Over the Last 2 Decades (1995-2014): Analysis of Current Data in the United States. Biological Psychiatry, 79, 613-619. doi:10.1016/j.biopsych.2016.01.004.

ElSohly, M. A., Ross, S. A., Mehmedic, Z., Arafat, R., Yi, B. y Banahan, B. F. (2000). Potency trends of delta9-THC and other cannabinoids in confiscated marijuana from 19801997. Journal of Forensic Sciences, 45, 24-30. Retrieved at http://www.ncbi.nlm.nih.gov/pubmed/10641915.

ElSohly, M. A. y Slade, D. (2005). Chemical constituents of marijuana: The complex mixture of natural cannabinoids. Life Sciences, 78, 539-548. doi:10.1016/j. lfs.2005.09.011.

Englund, A., Atakan, Z., Kralj, A., Tunstall, N., Murray, R. y Morrison, P. (2016). The effect of five day dosing with THCV on THC-induced cognitive, psychological and physiological effects in healthy male human vo- lunteers: A placebo-controlled, double-blind, crossover pilot trial. Journal of Psychopharmacology, 30, 140-151. doi:10.1177/0269881115615104.

Englund, A., Morrison, P. D., Nottage, J., Hague, D., Kane, F., Bonaccorso, S., ... Kapur, S. (2013). Cannabidiol inhibits THC-elicited paranoid symptoms and hippocampal-dependent memory impairment. Journal of Psychopharmacology, 27, 19-27. doi:10.1177/0269881112460109.

European Monitoring Centre for Drugs and Drug Addiction. (2012). Prevelance of Cannabis use in the European Union and Norway. Lisbon (Portugal). Retrieved at http://www.emcdda.europa.eu/publications/ thematic-papers / prevalence-daily-cannabis-use-european-union-and-norway.

European Monitoring Centre for Drugs and Drug Addiction. (2016). European Drug Report 2016: Trends and Developments. Luxembourg. Retrieved at http://www.emcdda.europa.eu/edr2016.

Folstein, M. F. y Luria, R. (1973). Reliability, validity, and clinical application of the visual analogue mood scale1. Psychological Medicine, 3, 479-483. doi:10.1017/ S0033291700054283.

Gaoni, Y. y Mechoulam, R. (1964). Isolation, Structure, and Partial Synthesis of an Active Constituent of Hashish. Journal of the American Chemical Society, 86, 1646-1647. doi:10.1021/ja01062a046.

Greenwald, M. K. y Stitzer, M. L. (2000). Antinociceptive, subjective and behavioral effects of smoked marijuana in humans. Drug and Alcohol Dependence, 59, 261-275. doi:10.1016/S0376-8716(99)00128-3.

Gual, A., Martos, A. R., Lligoña, A., Llopis, J. J., Lligona, A. y Llopis, J. J. (1999). Does the concept of a standard drink apply to viticultural societies? Alcohol and Alcoholism, 34, 153-160. doi:10.1093/alcalc/34.2.153.

Hall, W. (2009). The adverse health effects of cannabis use: What are they, and what are their implications for policy? International Journal of Drug Policy, 20, 458-466. doi:10.1016/j.drugpo.2009.02.013.

Hall, W. y Degenhardt, L. (2009). Adverse health effects of non-medical cannabis use. The Lancet, 374, 1383-1391. doi:10.1016/S0140-6736(09)61037-0.

Haney, M., Malcolm, R. J., Babalonis, S., Nuzzo, P. A., Cooper, Z. D., Bedi, G., ... Walsh, S. L. (2016). Oral Cannabidiol does not Alter the Subjective, Reinforcing or Cardiovascular Effects of Smoked Cannabis. Neuropsychopharmacology, 41, 1974-1982. doi:10.1038/npp.2015.367.

Hart, C., Ward, A., Haney, M., Comer, S., Foltin, R. y Fischman, M. (2002). Comparison of smoked marijuana and oral $\delta 9$-tetrahydrocannabinol in humans. Psychopharmacology, 164, 407-415. doi:10.1007/s00213002-1231-y.

Hazekamp, A., Bastola, K., Rashidi, H., Bender, J. y Verpoorte, R. (2007). Cannabis tea revisited: A systematic evaluation of the cannabinoid composition of cannabis tea. 
Journal of Ethnopharmacology, 113, 85-90. doi:10.1016/j. jep.2007.05.019.

Hollister, L. E. (1973). Cannabidiol and cannabinol in man. Experientia, 29, 825-826. doi:10.1007/BF01946311.

Hollister, L. E. (1974). Structure-Activity Relationships in Man of Cannabis Constituents, and Homologs and Metabolites of yDelta; ${ }^{9}$-Tetrahydrocannabinol. Pharmacology, 11, 3-11. doi:10.1159/000136462.

Hollister, L. E. (1987). Health aspects of cannabis. Journal of Ethnopharmacology, 19, 341-342. doi:10.1016/03788741 (87)90030-4.

Hollister, L. E. y Gillespie, H. (1975). Interactions in man of delta-9-tetrahydrocannabinol. II. Cannabinol and cannabidiol. Clinical Pharmacology and Therapeutics, 18, 80-3. Retrieved at http://www.ncbi.nlm.nih.gov/entrez/query.fcgi?cmd=Retrieveydb=PubMedydopt=Citationylist_uids=1097148.

Hollister, L. E. y Gillespie, H. K. (1973). Delta-8- and delta-9-tetrahydrocannabinol; Comparison in man by oral and intravenous administration. Clinical Pharmacology y Therapeutics, 14, 353-357. doi:10.1002/cpt1973143353.

Hunault, C. C., Mensinga, T. T., Böcker, K. B. E., Schipper, C. M. A., Kruidenier, M., Leenders, M. E. C., ... Meulenbelt, J. (2009). Cognitive and psychomotor effects in males after smoking a combination of tobacco and cannabis containing up to $69 \mathrm{mg}$ delta-9-tetrahydrocannabinol (THC). Psychopharmacology, 204, 85-94. doi:10.1007/ s00213-008-1440-0.

Hunault, C. C., Mensinga, T. T., de Vries, I., Kelholt-Dijkman, H. H., Hoek, J., Kruidenier, M., ... Meulenbelt, J. (2008). Delta-9-tetrahydrocannabinol (THC) serum concentrations and pharmacological effects in males after smoking a combination of tobacco and cannabis containing up to $69 \mathrm{mg}$ THC. Psychopharmacology, 201, 171-181. doi:10.1007/s00213-008-1260-2.

Ilan, A. B., Gevins, A., Coleman, M., ElSohly, M. A. y de Wit, H. (2005). Neurophysiological and subjective profile of marijuana with varying concentrations of cannabinoids. Behavioural Pharmacology, 16, 487-496. doi:10.1097/00008877-200509000-00023.

Karniol, I. G. y Carlini, E. A. (1973). Comparative Studies in Man and in Laboratory Animals on delta-8 and delta-9-trans-Tetrahydrocannabinol. Pharmacology, 9, 115-126.

Knight, G., Hansen, S., Connor, M., Poulsen, H., McGovern, C. y Stacey, J. (2010). The results of an experimental indoor hydroponic Cannabis growing study, using the "Screen of Green" (ScrOG) method-Yield, tetrahydrocannabinol (THC) and DNA analysis. Forensic Science International, 202, 36-44. doi:10.1016/j.forsciint.2010.04.022.

Lemberger, L., Martz, R., Rodda, B., Forney, R. y Rowe, H. (1973). Comparative Pharmacology of 89 Tetrahydrocannabinol and its Metabolite, 11-OH- 89 -
Tetrahydrocannabinol. Journal of Clinical Investigation, 52, 2411-2417. doi:10.1172/JCI107431.

Leweke, F. M., Mueller, J. K., Lange, B. y Rohleder, C. (2016). Therapeutic potential of cannabinoids in psychosis. Biological Psychiatry, 79, 604-612. doi:10.1016/j. biopsych.2015.11.018.

Liberati, A., Altman, D. G., Tetzlaff, J., Mulrow, C., Gøtzsche, P. C., Ioannidis, J. P. A., ... Moher, D. (2009). The PRISMA Statement for Reporting Systematic Reviews and Meta-Analyses of Studies That Evaluate Health Care Interventions: Explanation and Elaboration. PLoS Medicine, 6, e1000100. doi:10.1371/journal.pmed.1000100.

Martin-Santos, R., Crippa, J. A., Batalla, A., Bhattacharyya, S., Atakan, Z., Borgwardt, S., ... McGuire, P. K. (2012). Acute effects of a single, oral dose of d9-tetrahydrocannabinol (THC) and cannabidiol (CBD) administration in healthy volunteers. Current Pharmaceutical Design, 18, 4966-79. doi:18734286.

Mechoulam, R. (2005). Plant cannabinoids: a neglected pharmacological treasure trove. British Journal of Pharmacology, 146, 913-915. doi:10.1038/sj.bjp.0706415.

Mechoulam, R. y Hanuš, L. (2000). A historical overview of chemical research on cannabinoids. Chemistry and Physics of Lipids, 108, 1-13. doi:10.1016/S0009-3084(00)001845 .

Mehmedic, Z., Chandra, S., Slade, D., Denham, H., Foster, S., Patel, A. S., ... ElSohly, M. a. (2010). Potency trends of 89 -THC and other cannabinoids in confiscated cannabis preparations from 1993 to 2008. Journal of Forensic Sciences, 55, 1209-1217. doi:10.1111/j.15564029.2010.01441.x.

Morgan, C. J. A., Gardener, C., Schafer, G., Swan, S., Demarchi, C., Freeman, T. P., ... Curran, H. V. (2012). Sub-chronic impact of cannabinoids in street cannabis on cognition, psychotic-like symptoms and psychological well-being. Psychological Medicine, 42, 391-400. doi:10.1017/S0033291711001322.

Morgan, C. J. A., Schafer, G., Freeman, T. P. y Curran, H. V. (2010). Impact of cannabidiol on the acute memory and psychotomimetic effects of smoked cannabis: naturalistic study. The British Journal of Psychiatry, 197, 285290. doi:10.1192/bjp.bp.110.077503.

Niesink, R. J. M., Rigter, S., Koeter, M. W. y Brunt, T. M. (2015). Potency trends of $\delta 9$-tetrahydrocannabinol, cannabidiol and cannabinol in cannabis in the Netherlands: 2005-15. Addiction, 110, 1941-1950. doi:10.1111/ add.13082.

Observatorio Español de la Droga y las Toxicomanías. (2015). Alcohol, tabaco y drogas ilegales en España. Informe 2015.

Pérez-Reyes. (1973). A comparison of the Pharmacological Activity in Man of Intravenously Administered Delta-9-Tetrahydrocannabinol, Cannabinol, and Cannabidiol. Experientia, 1368-1369. 
Pijlman, F., Rigter, S., Hoek, J., Goldschmidt, H. y Niesink, R. (2005). Strong increase in total delta-THC in cannabis preparations sold in Dutch coffee shops. Addiction Biology, 10, 171-180. doi:10.1080/13556210500123217.

Plan Nacional sobre Drogas. (2016). Encuesta sobre el uso de drogas en enseñanzas secundarias (ESTUDES) 2014/5.

Potter, D. J., Clark, P. y Brown, M. B. (2008). Potency of $\delta 9$ -THC and Other Cannabinoids in Cannabis in England in 2005: Implications for Psychoactivity and Pharmacology. Journal of Forensic Sciences, 53, 90-94. doi:10.1111/ j.1556-4029.2007.00603.x.

Ramesh, D., Haney, M. y Cooper, Z. D. (2013). Marijuana's dose-dependent effects in daily marijuana smokers. Experimental and Clinical Psychopharmacology, 21, 287-293. doi:10.1037/a0033661.

Schaefer, C. F., Gunn, C. G. y Dubowski, K. M. (1977). Dose-Related heart-rate, perceptual, and decisional changes in man following marihuana smoking. Perceptual and Motor Skills, 44, 3-16. doi:10.2466/pms.1977.44.1.3.

Schwope, D. M., Bosker, W. M., Ramaekers, J. G., Gorelick, D. A. y Huestis, M. A. (2012). Psychomotor Performance, Subjective and Physiological Effects and Whole Blood 9-Tetrahydrocannabinol Concentrations in Heavy, Chronic Cannabis Smokers Following Acute Smoked Cannabis. Journal of Analytical Toxicology, 36, 405-412. doi:10.1093/jat/bks044.

Sharma, P., Murthy, P. y Bharath, M. M. S. (2012). Chemistry, metabolism, and toxicology of cannabis: clinical implications. Iranian Journal of Psychiatry, 7, 149-156.

Stockwell, T., Blaze-Temple, D. y Walker, C. (1991). The effect of "standard drink" labelling on the ability of drinkers to pour a "standard drink". Australian Journal of Public Health, 15, 56-63.

Tsumura, Y., Aoki, R., Tokieda, Y., Akutsu, M., Kawase, Y., Kataoka, T., ... Kurahashi, K. (2012). A survey of the potency of Japanese illicit cannabis in fiscal year 2010. Forensic Sci Int, 221, 77-83. doi:10.1016/j.forsciint.2012.04.005.

Turner, C. E. (1974). Active substances in marijuana. Archivos de Investigacion Médica, 5, 135-140. Retrieved at http://www.scopus.com/inward/record. url? eid=2-s 2.0-0016319961ypartnerID $=40 \mathrm{ymd} 5=-$ b11249203357e0c165116cf456425f6c

United Nations Office on Drugs and Crime. (2015). World Drug Report 2015. New York: United Nations. Retrieved at https://www.unodc.org/documents/wdr2015/ World_Drug_Report_2015.pdf

Urrútia, G. y Bonfill, X. (2010). Declaración PRISMA: una propuesta para mejorar la publicación de revisiones sistemáticas y metaanálisis. Medicina Clínica, 135, 507-511. doi:10.1016/j.medcli.2010.01.015.

Van der Kooy, F., Pomahacova, B. y Verpoorte, R. (2008). Cannabis Smoke Condensate I: The Effect of Different Preparation Methods on Tetrahydro- cannabinol Levels. Inhalation Toxicology, 20, 801-804. doi:10.1080/08958370802013559.

Van der Kooy, F., Pomahacova, B. y Verpoorte, R. (2009). Cannabis Smoke Condensate II: Influence of Tobacco on Tetrahydrocannabinol Levels. Inhalation Toxicology, 21, 87-90. doi:10.1080/08958370802187296.

Van der Pol, P., Liebregts, N., de Graaf, R., Korf, D. J., van den Brink, W. y van Laar, M. (2013). Validation of self-reported cannabis dose and potency: an ecological study. Addiction, 108, 1801-1808. doi:10.1111/add.12226.

Volkow, N. D., Baler, R. D., Compton, W. M. y Weiss, S. R. B. (2014). Adverse Health Effects of Marijuana Use. New England Journal of Medicine, 370, 2219-2227. doi:10.1056/NEJMra1402309.

Wachtel, S., ElSohly, M. A., Ross, S., Ambre, J. y Wit, H. de. (2002). Comparison of the subjective effects of $\delta$ 9 -tetrahydrocannabinol and marijuana in humans. Psychopharmacology, 161, 331-339. doi:10.1007/s00213-0021033-2.

World Health Organization. (2004). Neuroscience of Psychoactive Substance Use and Dependence. Addiction, 99, 1361-1362. doi:10.1111/j.1360-0443.2004.00906.x.

World Health Organization. (2016). The health and social effects of nonmedical cannabis use. Retrieved at http:/ / who.int/substance_abuse/publications/msbcannabis. pdf?ua $=1$.

Zhornitsky, S. y Potvin, S. (2012). Cannabidiol in Humans-The Quest for Therapeutic Targets. Pharmaceuticals, 5, 529-552. doi:10.3390/ph5050529.

Zuardi, A. W., Shirakawa, I., Finkelfarb, E. y Karniol, I. G. (1982). Action of cannabidiol on the anxiety and other effects produced by 9-THC in normal subjects. Psychopharmacology, 76, 245-250. doi:10.1007/BF00432554.

Zuurman, L., Roy, C., Schoemaker, R., Hazekamp, A., den Hartigh, J., Bender, J., ... van Gerven, J. (2008). Effect of intrapulmonary tetrahydrocannabinol administration in humans. Journal of Psychopharmacology, 22, 707-716. doi:10.1177/0269881108089581. 